\title{
Hyperglycemia in Critical Illness: Lessons From NICE-SUGAR
}

\author{
Alejandro A. Rabinstein
}

Published online: 5 June 2009

(C) Humana Press Inc. 2009

The parameters for optimal glucose control in critically ill patients have been a matter of heated debate for several years. The remarkable reduction in mortality observed in association with intensive insulin therapy in an unblinded, single-center study conducted in a surgical (predominantly cardiovascular) ICU in Leuven, Belgium [1] fueled an enormous drive to develop rigorous protocols for intensive insulin therapy to maintain strict normoglycemia (blood sugar $\leq 110 \mathrm{mg}$ per $\mathrm{dl}$ ). Yet, subsequent studies-including another from the medical ICU population of the same center in Leuven which reported improved morbidity but no improvement in mortality except for patients who stayed in the ICU for 3 days or more-[2] have shown much less favorable results. In fact, a meta-analysis published shortly before the publication of the NICE-SUGAR trial results concluded that intensive insulin therapy does not decrease mortality but does increase the risk of severe hypoglycemia in critically ill patients [3].

NICE-SUGAR was a parallel-group, randomized, controlled trial of medical and surgical patients admitted to the ICUs of 42 hospitals, mostly in academic centers [4]. Patients were eligible if they were expected to require ICU treatment for $\geq 3$ days. Treatment assignments were concealed before randomization but subsequent treatment administration was not blinded (an understandable limitation). Patients were randomized to an intensive glucose control target of 81-108 mg per dl (4.5-6.0 mmol per liter) or a conventional glucose control target of $180 \mathrm{mg}$ per $\mathrm{dl}$ or less $(10.0 \mathrm{mmol}$ per liter or less). A uniform web-based insulin protocol was strictly applied in all participating units.

A. A. Rabinstein ( $\square)$

Mayo Clinic, 200 First Street SW, Rochester, MN 55905, USA

e-mail: rabinstein.alejandro@mayo.edu
Mortality at 90 days, the primary endpoint of the study, was actually higher in patients assigned to the intensive glucose control group $(27.5 \%$ vs. $24.9 \%$ in the conventional control group; OR 1.14, 95\% CI 1.02-1.28; $P=0.02$ ). Mortality remained greater in the intensive control group after adjustment for predefined baseline risk factors. The difference was even more pronounced in surgical than in medical patients.

Insulin was administered to $97.2 \%$ of patients in the intensive control group but also to $69 \%$ of patients in the conventional control group. However, patients in the intensive control arm received a lot more insulin (mean of 50 vs. 17 units of insulin per day). Although patients in the intensive control arm often failed to achieve the strict prescribed target, there was adequate separation of the glycemias between the groups (mean time-weighted blood glucose level $115 \mathrm{mg}$ per $\mathrm{dl}$ in the intensive control versus $144 \mathrm{mg}$ per $\mathrm{dl}$ in the conventional control). Mean daily amount of nonprotein calories was modest and more than $70 \%$ of calories were provided enterally, according to widely accepted current practice guidelines.

There was no benefit from intensive insulin therapy across several predefined secondary and tertiary endpoints, such as length of stay in the ICU and the hospital, days on mechanical ventilation or renal replacement therapy, positive blood cultures, or blood transfusion. Severe hypoglycemia, defined as a blood sugar $\leq 40 \mathrm{mg}$ per dl ( $2.2 \mathrm{mmol}$ per liter), occurred in $6.8 \%$ of patients in the intensive control group versus $0.5 \%$ of patients in the conventional control group (OR 14.7). Still, these rates of hypoglycemia are lower than those noted in previous trials and the investigators reported that no episodes of hypoglycemia caused permanent sequelae.

The investigators did not include any information on baseline neurological disease or occurrence of neurological 
failure during the ICU admission. However, nearly a quarter of all proximate causes of death were classified as neurological and these neurological deaths were actually less common in the intensive control group, unlike cardiovascular deaths. This difference deserves further exploration. Furthermore, trauma patients were one of the only two subgroups (patients who received corticosteroids in the ICU being the other) of patients who tended to have lower mortality with intensive glucose control. Previous studies suggested a reduction in the risk of developing critical illness polyneuropathy associated with the use of intensive insulin therapy $[1,5]$. So far, no data from NICE-SUGAR on critical illness polyneuropathy has been reported.

Excellent editorials on this trial have been published which include thoughtful discussions on the possible reasons for the discrepancy of the results of NICE-SUGAR compared with those from the Leuven studies (single center biases and the practice of providing parenteral hyperalimentation with glucose loading at Leuven being the main proposed explanations) and the potential causes for the increased mortality observed in this multi-center study $[6,7]$. Although many questions still await answers, the results of NICE-SUGAR clearly indicate that intensive insulin therapy to achieve a target blood glucose below $110 \mathrm{mg}$ per $\mathrm{dl}$ should not be recommended for unselected ICU patients. However, as pointed out in the cited editorials, the findings of NICE-SUGAR should not be misinterpreted as indicating that careful monitoring and treatment of hyperglycemia is no longer advisable. Severe hyperglycemia and hypoglycemia are deleterious. Keeping blood sugar levels steadily between 140 and $180 \mathrm{mg}$ per dl using standardized protocols for insulin administration should be the objective for the care of critically ill patients based on current knowledge. Meanwhile, additional research should be conducted on the effects of hyperglycemia and the benefits of insulin therapy on neurological patients (such as patients with brain trauma, ischemic stroke patients undergoing reperfusion therapy, and patients with intracerebral hemorrhage) and neurological outcomes of critical systemic illness.

\section{References}

1. van den Berghe $G$, Wouters $P$, Weekers $F$, Verwaest $C$, Bruyninckx F, Schetz M, et al. Intensive insulin therapy in the critically ill patients. N Engl J Med. 2001;345:1359-67.

2. Van den Berghe G, Wilmer A, Hermans G, Meersseman W, Wouters PJ, Milants I, et al. Intensive insulin therapy in the medical ICU. N Engl J Med. 2006;354:449-61.

3. Wiener RS, Wiener DC, Larson RJ. Benefits and risks of tight glucose control in critically ill adults: a meta-analysis. JAMA. 2008;300:933-44.

4. The NICE-SUGAR Study Investigators. Intensive versus conventional glucose control in critically Ill patients. N Engl J Med. 2009; 360:1283-97.

5. Van den Berghe G, Schoonheydt K, Becx P, Bruyninckx F, Wouters PJ. Insulin therapy protects the central and peripheral nervous system of intensive care patients. Neurology. 2005;64: 1348-53.

6. Inzucchi SE, Siegel MD. Glucose control in the ICU—how tight is too tight? N Engl J Med. 2009;360:1346-9.

7. Bellomo R, Egi M. What is a NICE-SUGAR for patients in the intensive care unit? Mayo Clin Proc. 2009;84:400-2. 